\title{
Maximum Likelihood Based Estimation of Frequency and Phase Offset in DCT OFDM Systems under Non-Circular Transmissions: Algorithms, Analysis and Comparisons
}

\author{
Feifei Gao, Tao Cui, A. Nallanathan, Senior Member, IEEE, \\ and C. Tellambura, Senior Member, IEEE
}

\begin{abstract}
Recently, the advantages of the discrete cosine transform (DCT) based orthogonal frequency-division multiplexing (OFDM) have come to the light. We thus consider DCTOFDM with non-circular transmission (our results cover circular transmission as well) and present two blind joint maximumlikelihood frequency offset and phase offset estimators. Both our theoretical analysis and numerical comparisons reveal new advantages of DCT-OFDM over the traditional discrete Fourier transform (DFT) based OFDM. These advantages, as well as those already uncovered in the early works on DCT-OFDM, support the belief that DCT-OFDM is a promising multi-carrier modulation scheme.
\end{abstract}

Index Terms-Non-circular, carrier frequency offset, phase offset, DCT-OFDM, maximum likelihood.

\section{INTRODUCTION}

A LTHOUGH traditional discrete Fourier transform (DFT) based orthogonal frequency-division multiplexing (OFDM) [1] has succeeded in many applications and standards, another promising multi-carrier modulation known as discrete cosine transform (DCT) based OFDM has been proposed recently [2], where it is shown that DCT-OFDM can, sometimes, offer certain advantages over DFT-OFDM.

In multi-carrier modulation, a frequency offset, caused by oscillator mismatch or Doppler effects, destroys the subcarrier orthogonality and results in a substantial bit error rate (BER) degradation [3]. In the pioneering work [4], maximumlikelihood frequency offset estimation is developed for DFTOFDM. The algorithm exploits the cyclic prefix and is mainly proposed for additive white Gaussian noise (AWGN) channels. However, this method fails to provide phase offset estimation, a failure which is particularly significant when the channel is assumed to be AWGN, and the reason for which is that the transmitted symbols are complex circular random variables. Maximum-likelihood frequency offset estimation under noncircular transmission in DFT-OFDM has recently been studied

Paper approved by H. Minn, the Editor for Synchronization and Equalization of the IEEE Communications Society. Manuscript received July 18, 2006; revised November 22, 2006 and February 13, 2007.

F. Gao and A. Nallanathan were with the Department of Electrical \& Computer Engineering, National University of Singapore. F. Gao is with the Institute for Infocomm Research, 1 Fusionopolis Way, \#21-01 Connexis, Singapore 138632 (e-mail: feifeigao@ieee.org). A. Nallanathan is with the Division of Engineering, King's College London, United Kingdom (e-mail: nallanathan@ieee.org).

T. Cui is with the Department of Electrical Engineering, California Institute of Technology, Pasadena, CA 91125, USA (e-mail: taocui@ caltech.edu).

C. Tellambura is with the Department of Electrical \& Computer Engineering, University of Alberta, Edmonton, AB T6G 2V4, Canada (e-mail: chintha@ece.ualberta.ca).

Digital Object Identifier 10.1109/TCOMM.2008.060411. in [5], where it is shown that the estimator in [4] cannot provide the maximum-likelihood frequency offset estimate under non-circular transmission.

In this letter, we derive two blind joint maximum-likelihood frequency offset and phase offset estimators for DCT-OFDM for non-circular transmission. The anti-symmetry introduced in DCT-OFDM enables the full range of frequency offset estimation. As well, DCT-OFDM enables more accurate and more robust estimation than DFT-OFDM.

\section{Discrete Cosine Transform Based OFDM SYSTEM}

\section{A. System Model}

The $m$ th OFDM block in the frequency domain is represented as

$$
\mathbf{s}_{m}=\left[s_{m, 0}, s_{m, 1}, \ldots, s_{m, N-1}\right]^{T}, \quad m=0,1,2, \ldots,
$$

where $N$ is the number of subcarriers. The complex random variable $s_{m, i}$ is said to be non-circular at the order 2 , if $\mathrm{E}\left\{s_{m, i_{1}} s_{m, i_{2}}^{*}\right\}=E_{s} \delta_{i_{1} i_{2}}$, and $\mathrm{E}\left\{s_{m, i_{1}} s_{m, i_{2}}\right\}=E_{c} \delta_{i_{1} i_{2}}$, where $\delta_{a b}$ is the Kronecker delta function, $E_{s}$ is the average signal power, and $E_{c}$ may be a complex number with $\left|E_{c}\right| \leq E_{s}$. Here, symbols transmitted on different subcarriers are reasonably assumed independent from each other. The inverse DCT (IDCT) is performed on the $\mathbf{s}_{m}$, resulting in

$$
\mathbf{u}_{m}=\left[u_{m, 0}, u_{m, 1}, \ldots, u_{m, N-1}\right]^{T}=\mathbf{D}^{T} \mathbf{s}_{m},
$$

where $\mathbf{D}$ is the DCT matrix with the $(a, b)$ th entry

$$
[\mathbf{D}]_{a b}=\beta_{a} \sqrt{\frac{2}{N}} \cos \left(\frac{\pi(a-1)(2 b-1)}{2 N}\right), \quad a, b=1, \ldots, N .
$$

and

$$
\beta_{a}=\left\{\begin{array}{ll}
1 / \sqrt{2}, & a=1 \\
1, & \text { otherwise }
\end{array} .\right.
$$

If the number of subcarriers is sufficiently large, $u_{m, i}, i=$ $0, \ldots, N-1$ can be well approximated with a Gaussian distribution $^{1}$ [6]. It can be easily verified that,

$$
\mathrm{E}\left\{\mathbf{u}_{m} \mathbf{u}_{m}^{H}\right\}=E_{s} \mathbf{I}_{N}, \quad \mathrm{E}\left\{\mathbf{u}_{m} \mathbf{u}_{m}^{T}\right\}=E_{c} \mathbf{I}_{N} .
$$

Suppose that the maximum channel delay is smaller than $L T_{s}$, where $T_{s}$ is the sampling period. From [2], we know that in order to diagonalize the channel matrix for DCT-OFDM,

\footnotetext{
${ }^{1}$ It is not the true Gaussian. By normal approach, $N>4$ can well provide approximately Gaussian distributed variables.
} 
both the prefix and the suffix of length $\mu \geq\left\lceil\frac{L}{2}\right\rceil$ must be inserted into $\mathbf{u}_{m}$. Therefore, the effective OFDM block length is $(N+2 \mu) T_{s}$, and the transmitted symbols are represented by

$$
\mathbf{x}_{m}=\left[x_{m, 0}, x_{m, 1}, \ldots, x_{m, N+2 \mu-1}\right]^{T}=\mathbf{T}_{\mathrm{ps}} \mathbf{u}_{m},
$$

where

$$
\mathbf{T}_{\mathrm{ps}}=\left[\begin{array}{cc}
\mathbf{V}_{\mu} & \mathbf{0}_{\mu \times(N-\mu)} \\
& \mathbf{I}_{N} \\
\mathbf{0}_{\mu \times(N-\mu)} & \mathbf{V}_{\mu}
\end{array}\right]
$$

and $\mathbf{V}_{\mu}$ is the $\mu \times \mu$ anti-identity matrix. As in [4], we consider only the AWGN channel for the time being but still keep both the prefix and the suffix for estimation purposes. The baseband received signal can be expressed as

$$
\begin{aligned}
\mathbf{r}_{m} & =\left[r_{m, 0}, r_{m, 1}, \ldots, r_{m, N+2 \mu-1}\right]^{T} \\
& =e^{j 2 \pi m(N+2 \mu) \varepsilon_{0}+j \phi_{0}} \boldsymbol{\Gamma}\left(\varepsilon_{0}\right) \mathbf{x}_{m}+\mathbf{n}_{m},
\end{aligned}
$$

where $\varepsilon_{0}=f_{0} T_{s}$ is the frequency offset normalized by the sampling rate, and $\phi_{0}$ is the phase offset. Each element in $\mathbf{n}_{m}$ is an independent AWGN term with variance $E_{n}$, and $\boldsymbol{\Gamma}\left(\varepsilon_{0}\right)$ is the diagonal frequency offset matrix of the form

$$
\boldsymbol{\Gamma}\left(\varepsilon_{0}\right)=\operatorname{diag}\left\{1, e^{j 2 \pi \varepsilon_{0}}, \ldots, e^{j 2 \pi(N+2 \mu-1) \varepsilon_{0}}\right\} .
$$

\section{B. Joint frequency offset and phase offset Estimation}

We first consider the OFDM block with index number $m=$ 0 , and the index $m$ will be dropped for notational brevity. Define

$$
\begin{aligned}
& \mathbf{A}=E_{s} \mathbf{T}_{\mathrm{ps}} \mathbf{T}_{\mathrm{ps}}^{T}+E_{n} \mathbf{I}_{N+2 \mu}, \\
& \mathbf{B}=E_{c} \mathbf{T}_{\mathrm{ps}} \mathbf{T}_{\mathrm{ps}}^{T} .
\end{aligned}
$$

The covariance and the elliptic covariance of $\mathbf{r}$ are then $\boldsymbol{\Gamma}\left(\varepsilon_{0}\right) \mathbf{A} \boldsymbol{\Gamma}\left(\varepsilon_{0}\right)^{H}$ and $e^{j 2 \phi_{0}} \boldsymbol{\Gamma}\left(\varepsilon_{0}\right) \mathbf{B} \boldsymbol{\Gamma}\left(\varepsilon_{0}\right)$, respectively. Therefore, $\mathbf{r}$ is a non-circular Gaussian random vector and is characterized by the joint probability density function (PDF) [7]:

$$
\begin{aligned}
f\left(\mathbf{r}, \mathbf{r}^{*} \mid \varepsilon_{0}, \phi_{0}\right)= & \frac{1}{\pi^{N+L}(\operatorname{det}\{\mathbf{R}\})^{1 / 2}} \\
& \times \exp \left(-\frac{1}{2}\left[\mathbf{r}^{H}, \mathbf{r}^{T}\right] \mathbf{R}^{-1}\left[\begin{array}{c}
\mathbf{r} \\
\mathbf{r}^{*}
\end{array}\right]\right),
\end{aligned}
$$

where $\mathbf{R}$ is the covariance matrix of $\left[\mathbf{r}^{T}, \mathbf{r}^{H}\right]^{T}$, defined as

$$
\begin{aligned}
\mathbf{R}= & \mathrm{E}\left\{\left[\begin{array}{c}
\mathbf{r} \\
\mathbf{r}^{*}
\end{array}\right]\left[\mathbf{r}^{H}, \mathbf{r}^{T}\right]\right\} \\
= & \underbrace{\left[\begin{array}{cc}
e^{j \phi_{0}} \boldsymbol{\Gamma}\left(\varepsilon_{0}\right) & \mathbf{0} \\
\mathbf{0} & e^{-j \phi_{0}} \boldsymbol{\Gamma}\left(\varepsilon_{0}\right)^{*}
\end{array}\right]}_{\tilde{\boldsymbol{\Gamma}}} \underbrace{\left[\begin{array}{cc}
\mathbf{A} & \mathbf{B} \\
\mathbf{B}^{*} & \mathbf{A}^{*}
\end{array}\right]}_{\mathbf{R}_{s}} \\
& \times \underbrace{\left[\begin{array}{cc}
e^{-j \phi_{0}} \boldsymbol{\Gamma}\left(\varepsilon_{0}\right)^{*} & \mathbf{0} \\
\mathbf{0} & e^{j \phi_{0}} \boldsymbol{\Gamma}\left(\varepsilon_{0}\right)
\end{array}\right]}_{\tilde{\boldsymbol{\Gamma}}^{H}} .
\end{aligned}
$$

Using the inverse of a partitioned matrix [8], we obtain

$$
\mathbf{R}_{s}^{-1}=\left[\begin{array}{cc}
\mathbf{P} & -\mathbf{Q} \\
-\mathbf{Q}^{*} & \mathbf{P}^{*}
\end{array}\right],
$$

where $\mathbf{P}=\left(\mathbf{A}-\mathbf{B}^{H} \mathbf{A}^{-1} \mathbf{B}\right)^{-*}, \mathbf{Q}=\mathbf{A}^{-1} \mathbf{B} \mathbf{P}^{*}$, and $(\cdot)^{-*}$ denotes $\left((\cdot)^{*}\right)^{-1}$. Then $\mathbf{R}^{-1}=\tilde{\boldsymbol{\Gamma}} \mathbf{R}_{s}^{-1} \tilde{\boldsymbol{\Gamma}}^{H}$. From (12), we know that $\operatorname{det}\{\mathbf{R}\}=\operatorname{det}\left\{\mathbf{R}_{s}\right\}$. Therefore, $\operatorname{det}\{\mathbf{R}\}$ is a constant; it is sufficient to consider the log-likelihood function only. We hereby provides two different maximum-likelihood joint frequency offset and phase offset estimators.

1)\#MLE1 When frequency offset and phase offset are both considered as deterministic values, their maximum-likelihood estimates are

$$
\begin{aligned}
\left\{\hat{\varepsilon}_{0}, \hat{\phi}_{0}\right\} & =\arg \max _{\varepsilon, \phi} \log f\left(\mathbf{r}, \mathbf{r}^{*} \mid \varepsilon, \phi\right) \\
& =\arg \max _{\varepsilon, \phi}-\left[\mathbf{r}^{H}, \mathbf{r}^{T}\right] \mathbf{R}^{-1}\left[\begin{array}{c}
\mathbf{r} \\
\mathbf{r}^{*}
\end{array}\right],
\end{aligned}
$$

where $\varepsilon$ and $\phi$ are the dummy variables. Upon simplifying (14), we get the maximum-likelihood estimates of the frequency offset and phase offset as

$$
\left\{\hat{\varepsilon}_{0}, \hat{\phi}_{0}\right\}=\arg \max _{\varepsilon, \phi}-\mathbf{r}^{H} \mathbf{Y r}+\Re\left\{e^{-j 2 \phi} \mathbf{r}^{T} \mathbf{U r}\right\},
$$

where $\mathbf{Y}=\boldsymbol{\Gamma}(\varepsilon) \mathbf{P} \boldsymbol{\Gamma}(\varepsilon)^{*}$, and $\mathbf{U}=\boldsymbol{\Gamma}(\varepsilon)^{*} \mathbf{Q}^{*} \boldsymbol{\Gamma}(\varepsilon)^{*}$. Due to the special structure of $\mathbf{A}$ and $\mathbf{B}$, when $2 \mu<N,-\mathbf{r}^{H} \mathbf{Y r}$ can be rewritten as (16), where $\eta$ is some unimportant parameters unspecified for the concise of the paper, and $\mathbf{r}^{T} \mathbf{U r}$ can be expressed as in (17). Note that only the second term in (15) relates to the phase offset $\phi$. The phase offset can then be estimated via

$$
\hat{\phi}_{0}=\arg \max _{\phi} \Re\left\{e^{-j 2 \phi} \mathbf{r}^{T} \mathbf{U r}\right\}=\frac{1}{2} \angle \mathbf{r}^{T} \mathbf{U r},
$$

$$
\eta+\frac{2\left(2 E_{s}^{2}+E_{s} E_{n}-2\left|E_{c}\right|^{2}\right)}{E_{n}\left(4 E_{s}^{2}+4 E_{s} E_{n}+E_{n}^{2}-4\left|E_{c}\right|^{2}\right)} \sum_{i=0}^{\mu-1} \Re\left\{\left(r_{i}^{*} r_{2 \mu-i-1}+r_{i+N}^{*} r_{N+2 \mu-i-1}\right) e^{j 2 \pi(2 i-2 \mu+1) \varepsilon}\right\}
$$

$$
\begin{aligned}
& \frac{2 E_{c}^{*}}{4 E_{s}^{2}+4 E_{s} E_{n}+E_{n}^{2}-4\left|E_{c}\right|^{2}}\left(\sum_{i=0}^{\mu-1} r_{i}^{*} r_{2 \mu-i-1}^{*} e^{-j 2 \pi(2 \mu-1) \varepsilon}+\sum_{i=0}^{2 \mu-1} r_{i+N}^{*} r_{N+2 \mu-i-1}^{*} e^{-j 2 \pi(2 N+2 \mu-1) \varepsilon}\right) \\
& +\frac{E_{c}^{*}}{4 E_{s}^{2}+4 E_{s} E_{n}+E_{n}^{2}-4\left|E_{c}\right|^{2}}\left(\sum_{i=0}^{2 \mu-1} r_{i}^{*} r_{i}^{*} e^{-j 2 \pi(2 i) \varepsilon}+\sum_{i=N}^{N+2 \mu-1} r_{i}^{*} r_{i}^{*} e^{-j 2 \pi(2 i) \varepsilon}\right) \\
& +\frac{E_{c}^{*}}{E_{s}^{2}+2 E_{s} E_{n}+E_{n}^{2}-\left|E_{c}\right|^{2}} \sum_{i=2 \mu}^{N-1} r_{i}^{*} r_{i}^{*} e^{-j 2 \pi(2 i) \varepsilon} .
\end{aligned}
$$


where $\angle$ is the angle operation. Clearly, the estimation range of phase offset is $[-\pi / 2, \pi / 2)$. Substituting (18) back into (15), we find the frequency offset estimate to be

$$
\hat{\varepsilon}_{0}=\arg \max _{\varepsilon} \underbrace{-\mathbf{r}^{H} \mathbf{Y r}+\left|\mathbf{r}^{T} \mathbf{U r}\right|}_{g(\varepsilon)} .
$$

2)\#MLE2 When the statistics of either phase offset or frequency offset are known, maximum-likelihood estimation may be modified by using the marginal likelihood function. It is natural to assume that phase offset is a uniformly distributed random variable. From (18), we know that $f\left(\mathbf{r}, \mathbf{r}^{*} \mid \varepsilon, \phi\right)$ is a periodic function of $\phi$ with the period $\pi$. Therefore we assume that the phase offset $\phi_{0}$ is uniformly distributed over the region $[-\pi / 2, \pi / 2)^{2}$. The average of $f\left(\mathbf{r}, \mathbf{r}^{*} \mid \varepsilon, \phi\right)$ over $\phi$ gives the marginal likelihood function $f\left(\mathbf{r}, \mathbf{r}^{*} \mid \varepsilon\right)$ [9], which removes the likelihood dependence on $\phi$. This marginal likelihood function can thus be expressed as

$$
\begin{aligned}
f\left(\mathbf{r}, \mathbf{r}^{*} \mid \varepsilon\right) & =\mathrm{E}_{\phi}\left\{f\left(\mathbf{r}, \mathbf{r}^{*} \mid \varepsilon, \phi\right)\right\} \\
& =K \exp \left(-\mathbf{r}^{H} \mathbf{Y r}\right) \mathrm{E}_{\phi}\left\{\exp \left(\Re\left\{e^{-j 2 \phi} \mathbf{r}^{T} \mathbf{U r}\right\}\right)\right\} \\
& =\frac{K}{\pi} \exp \left(-\mathbf{r}^{H} \mathbf{Y r}\right) \int_{-\pi / 2}^{\pi / 2} \exp \left(\Re\left\{e^{-j 2 \phi} \mathbf{r}^{T} \mathbf{U} \mathbf{r}\right\}\right) d \phi \\
& =K \exp \left(-\mathbf{r}^{H} \mathbf{Y r}\right) I_{0}\left(\left|\mathbf{r}^{T} \mathbf{U} \mathbf{r}\right|\right) .
\end{aligned}
$$

where $K=\left(\pi^{N+L}(\operatorname{det}\{\mathbf{R}\})^{1 / 2}\right)^{-1}$ is a constant factor, and $I_{0}(\cdot)$ is the zeroth order modified Bessel function of the first kind. Then, the frequency offset $\varepsilon_{0}$ should be estimated from

$$
\begin{aligned}
\hat{\varepsilon}_{0} & =\arg \max _{\varepsilon} f\left(\mathbf{r}, \mathbf{r}^{*} \mid \varepsilon\right) \\
& =\arg \max _{\varepsilon} \exp \left(-\mathbf{r}^{H} \mathbf{Y r}\right) I_{0}\left(\left|\mathbf{r}^{T} \mathbf{U r}\right|\right) .
\end{aligned}
$$

For large $\left|\mathbf{r}^{T} \mathbf{U r}\right|$, the zeroth order modified Bessel function approximates as $I_{0}\left(\left|\mathbf{r}^{T} \mathbf{U r}\right|\right) \approx \frac{e^{\left|\mathbf{r}^{T} \mathbf{U r}\right|}}{\sqrt{2 \pi\left|\mathbf{r}^{T} \mathbf{U r}\right|}}$. Thus for high signal-to-noise ratio (SNR), $f\left(\mathbf{r}, \mathbf{r}^{*} \mid \varepsilon\right)$ can be rewritten as

$$
f\left(\mathbf{r}, \mathbf{r}^{*} \mid \varepsilon\right) \approx K \frac{\exp \left(-\mathbf{r}^{H} \mathbf{Y r}+\left|\mathbf{r}^{T} \mathbf{U} \mathbf{r}\right|\right)}{\sqrt{2 \pi}\left|\mathbf{r}^{T} \mathbf{U} \mathbf{r}\right|^{1 / 2}} .
$$

Unfortunately, a reasonable assumption on the distribution of the frequency offset $\varepsilon_{0}$ is not available in the literature ${ }^{3}$. Therefore, we are unable to average $f\left(\mathbf{r}, \mathbf{r}^{*} \mid \varepsilon, \phi\right)$ over $\varepsilon$ to obtain $f\left(\mathbf{r}, \mathbf{r}^{*} \mid \phi\right)$. However, since the PDF of $\phi$ is known, we may consider the maximum a posteriori (MAP) approach to detect $\phi_{0}$. From the Bayes rule, we have

$f\left(\phi \mid \mathbf{r}, \mathbf{r}^{*}, \hat{\varepsilon}_{0}\right)=\frac{f\left(\mathbf{r}, \mathbf{r}^{*} \mid \hat{\varepsilon}_{0}, \phi\right) f\left(\hat{\varepsilon}_{0}, \phi\right)}{f\left(\mathbf{r}, \mathbf{r}^{*} \mid \hat{\varepsilon}_{0}\right) f\left(\hat{\varepsilon}_{0}\right)}=\frac{f\left(\mathbf{r}, \mathbf{r}^{*} \mid \hat{\varepsilon}_{0}, \phi\right)}{\pi f\left(\mathbf{r}, \mathbf{r}^{*} \mid \hat{\varepsilon}_{0}\right)}$.

Note that the denominator is independent from $\phi$. Then $\phi_{0}$ is estimated from

$$
\hat{\phi}_{0}=\arg \max _{\phi} f\left(\mathbf{r}, \mathbf{r}^{*} \mid \hat{\varepsilon}_{0}, \phi\right),
$$

This gives exactly the same result as (18).

\footnotetext{
${ }^{2}$ Normally the phase offset should be uniformly distributed over the region $[-\pi, \pi)$. However, if $\left|\phi_{0}\right|>\pi / 2$, then only $\left(\phi_{0} \bmod \pi\right)-\pi / 2$ can be found by the proposed estimator. Therefore the effective phase offset can reasonably be assumed to be uniformly distributed over the region $[-\pi / 2, \pi / 2)$.

${ }^{3}$ If frequency offset is assumed to be uniformly distributed in $[-0.5,0.5]$, then similar process can be conducted.
}

\section{Discussion}

Since MLE2 includes a complicated Bessel function, our discussion mainly focuses on MLE1. Nevertheless, as shown by the simulations, both these two estimators perform identically within the regular SNR region.

1) Estimation Range: Thanks to the anti-symmetric property of the prefix and the suffix introduced in [2], $-\mathbf{r}^{H} \mathbf{Y r}$ contains the term $e^{j 2 \pi \varepsilon}$; hence, MLE1 provides full-range frequency offset estimation $\left(\varepsilon_{0} \in[-0.5,0.5]\right)$ as long as a) $\mu>0$; b) $r_{\mu-1}^{*} r_{\mu}+r_{\mu-1+N}^{*} r_{\mu+N} \neq 0$. Note that condition b) is equivalent to $\left(\left|u_{0}\right|^{2}+\left|u_{N-1}\right|^{2}+n_{\mu-1}^{*} n_{\mu}+n_{\mu-1+N}^{*} n_{\mu+N}\right) \neq$ 0 . Since $n_{i}$ are continuous Gaussian random variables, condition b) holds with probability one in the noisy case. If the prefix and suffix length $\mu=0$, then, the estimator $g(\varepsilon)$ contains only the third term in $\mathbf{r}^{T} \mathbf{U r}$, see (17):

$$
g(\varepsilon) \Leftarrow \frac{E_{c}^{*}}{E_{s}^{2}+2 E_{s} E_{n}+E_{n}^{2}-\left|E_{c}\right|^{2}}\left|\sum_{i=0}^{N-1} r_{i}^{*} r_{i}^{*} e^{-j 2 \pi(2 i) \varepsilon}\right| \text {. }
$$

As long as $r_{1}^{*} r_{1} \neq 0$, the exponential with the smallest order is $r_{1}^{*} r_{1}^{*} e^{-j 4 \pi \varepsilon}$, the period on $\varepsilon$ decreases to $1 / 2$. Hence, the frequency offset estimation range reduces to $[-0.25,0.25]$. By the same reason, this half frequency offset estimation range holds with probability one. Note that, even when the prefix and suffix lengths are zero $(\mu=0)$, the frequency offset estimation range is still $N / 2$ times larger than that allowed by DFTOFDM [4], [5].

2) Circular Transmissions: Maximum-likelihood frequency offset estimates for circular transmissions can be obtained by simply setting the elliptic variance of $s_{i}$ as zero. In this case, $\mathbf{B}$ and $\mathbf{U}$ become zero matrices. Hence, the phase offset cannot be estimated ${ }^{4}$. However, the frequency offset would still be estimated from

$$
\begin{aligned}
\hat{\varepsilon}_{0}=\arg \max _{\varepsilon}-\mathbf{r}^{H} \mathbf{Y r} & \\
=\arg \max _{\varepsilon} \sum_{i=0}^{\mu-1} \Re\left\{\left(r_{i}^{*} r_{2 \mu-i-1}\right.\right. & \left.+r_{i+N}^{*} r_{N+2 \mu-i-1}\right) \\
& \left.\times e^{j 2 \pi(2 i-2 \mu+1) \varepsilon}\right\} .
\end{aligned}
$$

Note that, $\mu>0$ is required, otherwise $-\mathbf{r}^{H} \mathbf{Y r}$ is independent of $\varepsilon$, as explained previously.

3) frequency offset Tracking: Tracking the residual frequency offset and phase offset is also possible by considering the subsequent blocks $(m>0)$. Namely, for each new received block with $m>0$, we can estimate new frequency offset and phase offset corresponding to this specific block. If the $m$ th OFDM block is considered, we may regard $2 \pi m(N+2 \mu) \varepsilon_{0}+\phi_{0}$ as the equivalent phase offset and carry on the similar steps to estimate frequency offset and phase offset. The phase offset estimator (18) could then be rewritten as

$$
\begin{aligned}
\hat{\phi}_{0} & =\arg \max _{\phi} \Re\left\{e^{-j 2(2 \pi m(N+2 \mu) \varepsilon+\phi)} \mathbf{r}^{T} \mathbf{U r}\right\} \\
& =\frac{1}{2} \angle\left(e^{-j 4 \pi m(N+2 \mu) \varepsilon} \mathbf{r}^{T} \mathbf{U r}\right) .
\end{aligned}
$$

\footnotetext{
${ }^{4}$ The phase offset could be estimated if some pilots are inserted into each OFDM block. Since we are considering the estimations in a blind way, we do not assume any pilot here.
} 


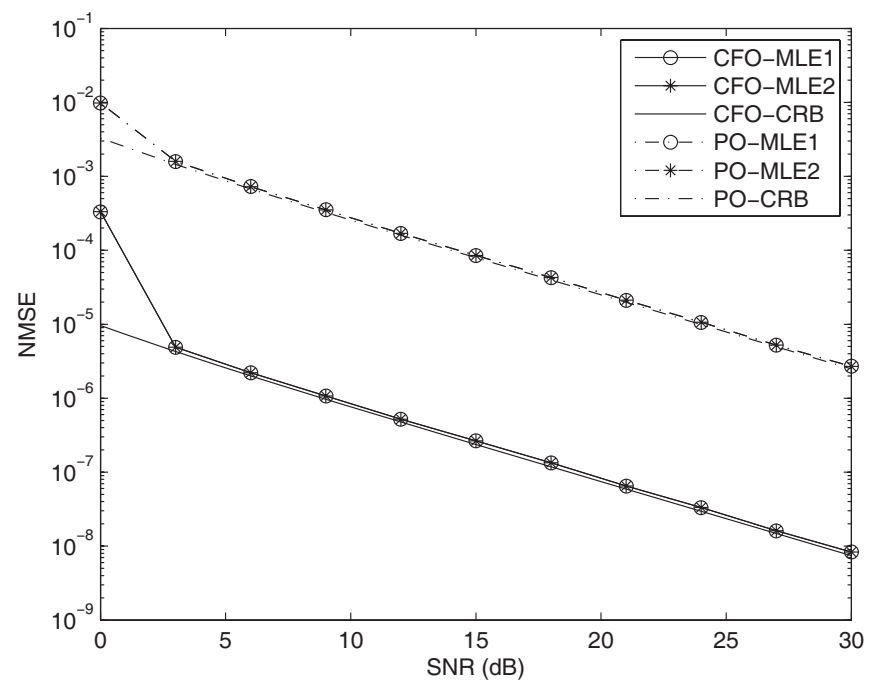

Fig. 1. Performance comparison between the MLE1 and MLE2 in DCTOFDM.

Nevertheless, the maximum-likelihood frequency offset estimator (19) remains unchanged.

\section{Simulation Results}

In this section, we examine the proposed estimators under various scenarios. We consider $N=64$ subcarriers and apply $M_{q}=500$ Monte Carlo runs are conducted to average the simulation results. All symbols are assumed to be obtained from a BPSK constellation, namely, $E_{s}=E_{c}$.

1) Performance comparison between the MLE1 and MLE2. The normalized frequency offset is chosen as large as $\varepsilon_{0}=0.2$ (more than 12 subcarrier spacings) and phase offset is set as $\pi / 3$. We first compare our two estimators when the length of both the prefix and suffix is 8 . The frequency offset and phase offset NMSEs versus SNR performances are shown in Fig. 1. The Cramér-Rao bounds (CRB) [10] for frequency offset and phase offset estimations are displayed as well. Not only do both the estimators provide the same performance, but they also agree well with the CRB.

2). Performance comparison for different $\mu$. We now examine the performance as a function of the length of the prefix and the suffix. Since the previous experiment shows that both proposed estimators perform identically, we only consider MLE1. The frequency offset and phase offset NMSEs versus SNR, for $\mu=0,2,4,8$ are drawn in Fig. 2 with their corresponding CRBs. Clearly, the frequency offset estimation accuracy improves when the length $\mu$ is increased, about $4 \mathrm{~dB}$ when $\mu$ is increased from 0 to 8 . However, the phase offset estimation accuracy is not sensitive to the length of the prefix and suffix.

3) Comparing frequency offset estimation of circular and non-circular estimators. We now illustrate the performance of the estimator (26) for BPSK. Since $\mu$ in (26) must be greater than zero, we select three values as $\mu=2,4,8$. Fig. 3 shows frequency offset NMSEs versus SNR for both the estimator (26) and the maximum-likelihood estimator (19). The performance loss is significant if one ignores the noncircularity of the transmitted symbols and applies the circular

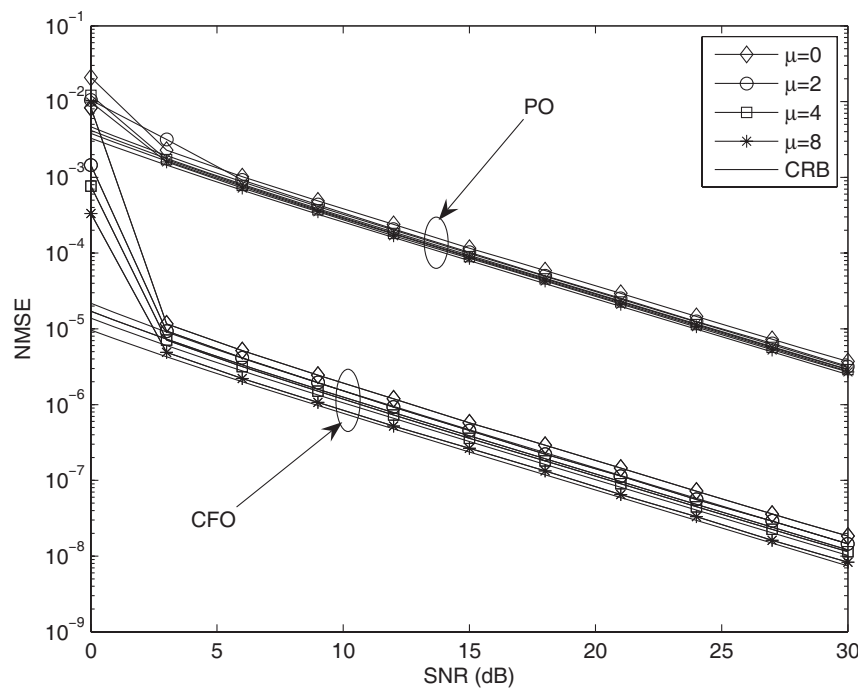

Fig. 2. Performance comparison of the frequency offset/phase offset estimations for different $\mu$ in DCT-OFDM.

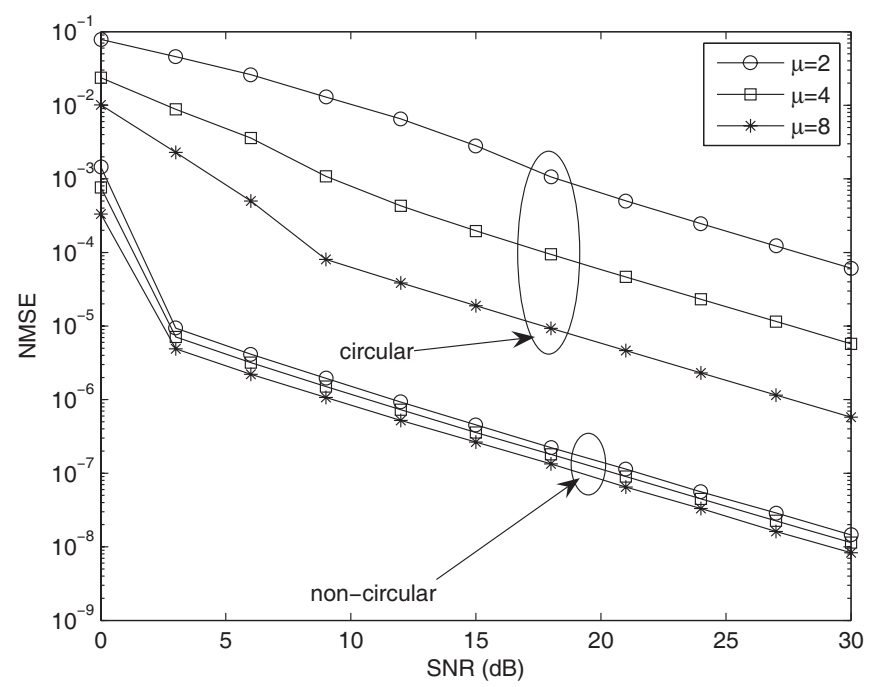

Fig. 3. Comparing frequency offset estimation of circular and non-circular estimators in DCT OFDM system.

estimator (26) directly. Especially when the length of the prefix and suffix is small, the performance loss is significantly large. Therefore, the DCT-OFDM performance depends on whether the transmission is circular or non-circular, and ignoring the non-circularity, if any, greatly degrades the performance. Consequently, it is quite important to develop DCT-OFDM estimators that takes the non-circularity into consideration.

4) Comparison Between DCT-OFDM and DFT-OFDM. We now illustrate how the performance of these two OFDM systems differs for the non-circular transmission considered. Since DFT-OFDM is also considered, the parameters change to $\varepsilon_{0}=0.15 / N$, and $\phi_{0}=\pi / 3$. For a fair comparison, the total amount of redundancy added to one OFDM block is fixed, namely, $L=2 \mu$. The frequency offset NMSEs versus SNR are shown in Fig. 4. In each figure, the solid lines from up to down represent DCT-OFDM with $\mu=2,4,8$, whereas the dashed lines from up to down represent DFT-OFDM with $L=4,8,16$. Two phenomena are observed. 1) DCT-OFDM performs better than DFT-OFDM, especially when the amount 


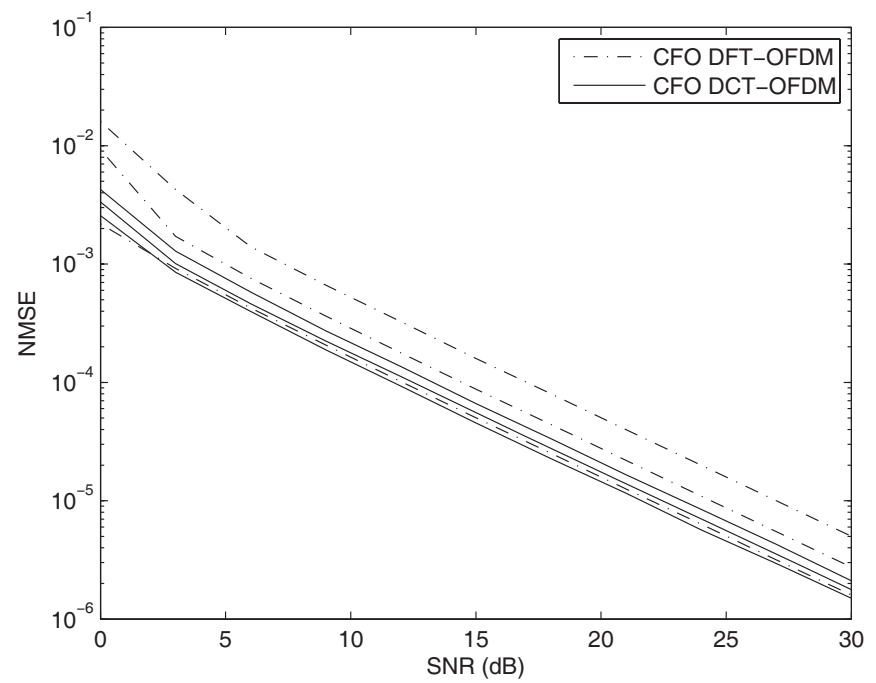

Fig. 4. Comparison between DCT-OFDM and DFT-OFDM under noncircular transmission, frequency offset estimation.

of the redundancy added to one OFDM block is small. 2) The performance of DCT-OFDM varies slightly when the prefix and suffix length $\mu$ changes. However, the performance of DFT-OFDM is highly sensitive to the suffix length $L$. Thus frequency offset estimation in DCT-OFDM is more robust to the amount of the redundancy than that in DFT-OFDM. Similar phenomenon was observed for phase offset estimation. However, due to the page limit, we omit the discussion here.

\section{CONCLUSIONS}

In this letter, we considered non-circular transmission of DCT-OFDM and proposed two maximum-likelihood joint frequency offset and phase offset estimators. The phase offset can be estimated only if the transmitted symbols are noncircular. In addition to those mentioned in [2], we found several new advantages of DCT-OFDM over DFT-OFDM. First, with DCT-OFDM, the frequency offset estimation range increases from only one subcarrier spacing to its maximum. Second, the frequency offset and phase offset estimation is not only more accurate, but also more robust to the amount of the redundancy per block.

\section{ACKNOWLEDGMENT}

The authors would like to thank the anonymous reviewers for their valuable comments that greatly improved the presentation of this letter.

\section{REFERENCES}

[1] J. A. C. Bingham, "Multicarrier modulation for data transmission: an idea whose time has come," IEEE Commun. Mag., vol. 28, pp. 5-14, May 1990.

[2] N. Al-Dhahir, H. Minn, and S. Satish, "Optimum DCT-based multicarrier transceivers for frequency-selective channels," IEEE Trans. Commun., vol. 54, pp. 911-921, May 2006.

[3] T. Pollet, M. van Bladel, and M. Moeneclaey, "BER sensitivity of OFDM systems to carrier frequency offset and Wiener phase noise," IEEE Trans. Commun., vol. 43, pp. 191-193, Feb. 1995.

[4] J. J. van de Beek, M. Sandell, and P. O. Borjesson, "ML estimation of time and frequency offset in OFDM systems," IEEE Trans. Signal Processing, vol. 45, pp. 1800-1805, July 1997.

[5] T. Fusco and M. Tanda, "ML-based symbol timing and frequency offset estimation for OFDM systems with noncircular transmissions," IEEE Trans. Signal Processing, vol. 54, pp. 3527-2541, Sept. 2006.

[6] E. Y. Lam and J. W. Goodman, "A mathematical analysis of the DCT coefficient distributions for images," IEEE Trans. Image Processing, vol. 9, pp. 1661-1666, Oct. 2000.

[7] B. Picinbono, "Second order complex random vectors and normal distributions," IEEE Trans. Signal Processing, vol. 44, pp. 2637-2640, Oct. 1996.

[8] P. Stoica and R. Moses, Introduction to Spectral Analysis. Upper Saddle River, NJ: Prentice-Hall 1997.

[9] H. L. van Trees, Detection, Estimation, and Modulation Theory. Weiley Interscience, 2001.

[10] J.-P. Delmas and H. Abeida, "Stochastic Cramer-Rao bound for noncircular signals with application to DOA estimation," IEEE Trans. Signal Processing, vol. 52, pp. 3192-3199, Nov. 2004. 